\title{
openheart Functional classification discordance in intermediate coronary stenoses between fractional flow reserve and angiography- based quantitative flow ratio
}

\author{
Yoshinori Kanno, ${ }^{1}$ Masahiro Hoshino (1) , ${ }^{1}$ Rikuta Hamaya, ${ }^{1}$ Tomoyo Sugiyama, ${ }^{1}$ \\ Yoshihisa Kanaji, ${ }^{1}$ Eisuke Usui, ${ }^{1}$ Masao Yamaguchi, ${ }^{1}$ Masahiro Hada, ${ }^{1}$ \\ Hiroaki Ohya, ${ }^{1}$ Yohei Sumino, ${ }^{1}$ Hidenori Hirano, ${ }^{1}$ Haruhito Yuki, ${ }^{1}$ Tomoki Horie, ${ }^{1}$ \\ Tadashi Murai, ${ }^{1}$ Tetsumin Lee, ${ }^{2}$ Taishi Yonetsu (D) , ${ }^{2}$ Tsunekazu Kakuta (D) ${ }^{1}$
}

\begin{abstract}
- Additional material is published online only. To view please visit the journal online (http://dx.doi.org/10.1136/ openhrt-2019-001179).
\end{abstract}

To cite: Kanno Y, Hoshino M, Hamaya $\mathrm{R}$, et al. Functional classification discordance in intermediate coronary stenoses between fractional flow reserve and angiography-based quantitative flow ratio. Open Heart 2020;7:e001179. doi:10.1136/

openhrt-2019-001179

Received 5 October 2019 Revised 19 November 2019 Accepted 17 December 2019

Check for updates

(C) Author(s) (or their employer(s)) 2020. Re-use permitted under CC BY-NC. No commercial re-use. See rights and permissions. Published by BMJ.

${ }^{1}$ Department of Cardiovascular Medicine, Tsuchiura Kyodo General Hospital, Tsuchiura, Japan

${ }^{2}$ Department of Interventional Cardiology, Tokyo Medical and Dental University, Tokyo, Japan

Correspondence to

Tsunekazu Kakuta; kaz@joy. email.ne.jp

\section{ABSTRACT}

Background Measurement of the contrast-flow quantitative flow ratio (cQFR) is a novel method for rapid computational estimation of fractional flow reserve (FFR). Discordance between FFR and cQFR has not been completely characterised.

Methods We performed a post-hoc analysis of 504 vessels with angiographically intermediate stenosis in 504 patients who underwent measurement of FFR, coronary flow reserve (CFR), the index of microcirculatory resistance (IMR) and Duke jeopardy score.

Results In total, 396 (78.6\%) and 108 (21.4\%) lesions showed concordant and discordant FFR and CQFR functional classifications, respectively. Among lesions with a reduced FFR (FFR+), those with a preserved CQFR (CQFR-) showed significantly lower IMR, shorter mean transit time (Tmn), shorter lesion length (all, $p<0.01$ ) and similar CFR and Duke jeopardy scores compared with lesions showing a reduced cQFR (cQFR+). Furthermore, lesions with FFR+ and cQFRhad significantly lower IMR and shorter Tmn compared with lesions showing a preserved FFR (FFR-) and CQFR+. Of note, in CQFR+ lesions, higher IMR lesions were associated with decreased diagnostic accuracy (high-IMR; 63.0\% and low-IMR; 75.8\%, p<0.01). In contrast, in CQFR- lesions, lower IMR lesions was associated with decreased diagnostic accuracy (high-IMR group; 96.8\% and low-IMR group; $80.0 \%, p<0.01)$. Notably, in total, 31 territories $(6.2 \%$; jump out' group) had an FFR above the upper limit of the grey zone $(>0.80)$ and a cQFR below the lower limit $(\leq 0.75)$. In contrast five territories (1.0\%; 'jump in' group) exhibited opposite results (FFR of $\leq 0.75$ and cQFR of $>0.80$ ). The 'jump out' territories showed significantly higher IMR values than 'jump in' territories $(p<0.01)$

Conclusions FFR- with CQFR+ is associated with increased microvascular resistance, and FFR+ with CQFR- showed preservation of microvascular function with high coronary flow. Microvascular function affected diagnostic performance of CQFR in relation to functional stenosis significance.

\section{INTRODUCTION}

Measurement of the fractional flow reserve (FFR) has become a standard practice for

\section{Key questions}

What is already known about this subject?

- Measurement of the contrast-flow quantitative flow ratio (CQFR) is a novel method for rapid computational estimation of the fractional flow reserve (FFR) derived from three-dimensional quantitative coronary angiography. The cQFR has been developed and validated to estimate the FFR and showed high accuracy for estimating the FFR in previous studies.

What does this study add?

- Despite the high agreement rate, discrepancy between the FFR-guided and CQFR-guided decisionmakings was documented in $20 \%$ of patients when the cut-off value of 0.80 was used. We demonstrated that microvascular resistance influenced the discordant functional assessment between the cQFR and FFR, whereas no significant difference was found in the subtended cardiac mass.

How might this impact on clinical practice?

- Larger prospective studies are warranted to clarify the potential utility of CQFR-guided versus FFR-guided decision-making, especially in the identification of microvascular dysfunction and coronary flow.

revascularisation decision-making in evaluating the functional significance of angiographically intermediate epicardial coronary stenosis. ${ }^{12}$ Despite the supporting evidence for the FFR, adoption and penetration of FFR measurements has been limited. ${ }^{34}$ This may be related to the cost of the pressure wire and limitations associated with induction of hyperaemia. A practical tool that facilitates FFR calculation without a pressure wire or hyperaemic induction could supplement FFR-guided revascularisation decisionmaking. Invasive quantitative coronary angiography (QCA)-based computational FFR by 
various methods has been reported. The contrast-flow quantitative flow ratio (cQFR) was recently developed and validated to estimate the FFR. ${ }^{56}$ The Functional Assessment by Various Flow Reconstructions (FAVOR) pilot study showed the potential of QFR calculation in identifying the functional stenosis by core laboratory-based calculations. ${ }^{5}$ In the Functional Diagnostic Accuracy of Quantitative Flow Ratio in Online Assessment of Coronary Stenosis study (FAVOR China II study), the cQFR was compared with the FFR and exhibited an overall diagnostic accuracy of more than $90 \%$ for detecting an FFR of $\leq 0.80 .{ }^{7}$ Subsequent studies showed similar results for online assessment of intermediate stenosis or the use of myocardial perfusion imaging as the reference standard to define myocardial ischaemia. ${ }^{78}$ Despite the high agreement rate, the FFR and cQFR show a discrepancy of $10 \%-20 \%$ when an FFR cut-off value of 0.80 is used to guide revascularisation. ${ }^{59}$ The cQFR is calculated based on a contrast-flow hyperaemic flow velocity model derived from coronary angiography (CAG) without pharmacologically induced hyperaemia. The contrast-flow is converted to virtual hyperaemic flow obtained from previous studies and applied as if vasodilator drugs had been administered. ${ }^{5}{ }^{6}$ We hypothesised that discordant decision-making between FFR and cQFR might be related to the difference in the individual microvascular function represented by the index of microcirculatory resistance (IMR), both resulting in the decreased diagnostic accuracy in lesions with increased microvascular resistance as reported,$^{10}$ and also resulting in lower agreement in lesions with decreased microvascular resistance. Furthermore, because the IMR is reportedly inversely associated with the amount of myocardium subtended by a coronary stenosis, we also evaluated the influence of myocardial mass subtended by an intermediate stenosis and other clinical factors on the discordant decisions between these two measures. ${ }^{11}$

\section{MATERIALS AND METHODS \\ Study population}

This post-hoc analysis included patients from the data sets of previous studies from our institution performed from January 2011 to June 2017. ${ }^{12}$ All screened patients had suspected or known stable coronary artery disease and underwent invasive CAG with physiological lesion assessment using a pressure-temperature sensor-tipped guide wire (PressureWire; St. Jude Medical, St. Paul, MN, USA). Lesions showing an angiographically visible stenosis of $30 \%-80 \%$ by visual estimation were included. In multivessel coronary artery disease, a single vessel with the lowest FFR value was included for the current analysis. The exclusion criteria were presented in an online supplementary file methods 1 . This study was performed in compliance with the Declaration of Helsinki for investigation in human beings. The study protocol was approved by the institutional review board, and all patients provided written informed consent for enrolment in the institutional database for potential future investigations. All patient data and procedural details were obtained from the patients' medical records.

\section{Coronary catheterisation and physiological measurements}

Coronary catheterisation and physiological measurements were performed as previously described, ${ }^{13} 14$ and shown in an online supplementary file methods 2.

\section{Computation of cQFR}

Computation of the cQFR was performed offline using a proprietary software package (QAngio XA 3D Research Edition 1.1; Medis Specials BV, Leiden, The Netherlands). First, two angiographic images at least $25^{\circ}$ apart were transferred to a computer equipped with a QFR system, and three-dimensional reconstruction of the interrogated vessel was performed with a few steps with observer interaction as previously described. ${ }^{56}$ The concept and basis of the underlying principles of QFR have been described elsewhere. ${ }^{56}$ The contrast-flow model uses a Thrombolysis in Myocardial Infarction (TIMI) frame count model to derive the contrast flow velocity from CAG. Frame count analysis was separately performed on the two angiographic projections without pharmacologically induced hyperaemia. Previous studies reported that cQFR showed a better diagnostic accuracy compared with fixed-flow QFR. Thus, we used cQFR values in this study. The cQFR value was obtained at the position where the FFR value was measured by referring to the fluoroscopic image for the sensor position documentation. cQFR analysis was performed by two well-trained investigators (YKanno and MHo) blinded to the FFR data.

\section{Reference standard of functionally significant stenosis and discordance of decision-making between FFR and cQFR}

The pressure-temperature sensor-tipped wire-derived FFR was used as the reference standard, and the diagnostic accuracy of the cQFR was assessed. The ischaemic threshold was defined as 0.80 for both measures. Furthermore, we investigated the occurrence and frequency of functional assessment that jumped beyond the grey zone (0.75-0.80) into the opposite decision-making zone between FFR-guided and cQFR-guided decisionmaking. The 'jump out' group was defined as a cQFR of $\leq 0.75$ with an FFR of $>0.80$, and the 'jump in' group was defined as a cQFR of $>0.80$ with an FFR of $\leq 0.75$. Representative cases in the 'jump out' and 'jump in' groups are shown in figure 1 . We assessed whether hyperaemic microvascular resistance represented by the IMR and the cardiac mass subtended by the lesion described by the Duke jeopardy score were associated with discordance between FFR-based and cQFR-based decision-making. We also compared cQFR-based decision-making with QCA assessment by $50 \%$ diameter stenosis.

\section{Statistical analysis}

The statistical analysis was performed using the SPSS V.23.0. Categorical data are expressed as absolute frequencies and percentages, and were compared using 
A
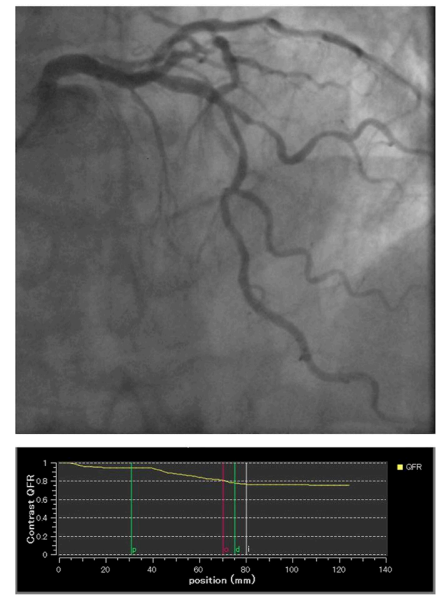

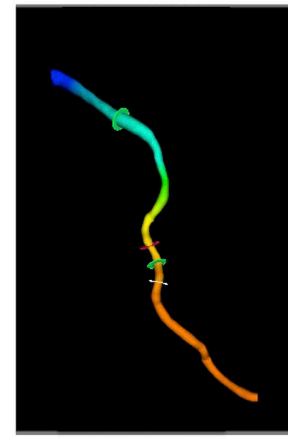

FFR 0.83

cQFR 0.75

IMR 41.8
B

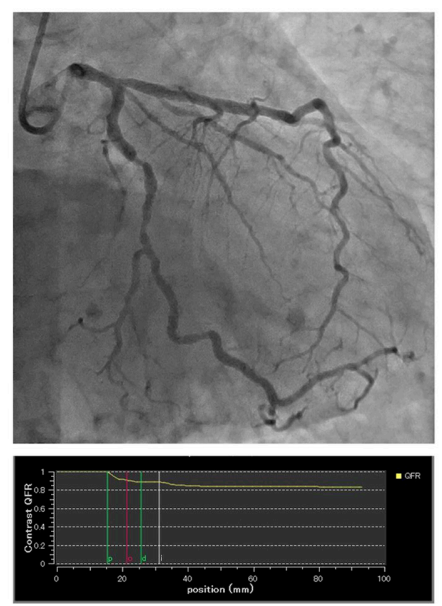

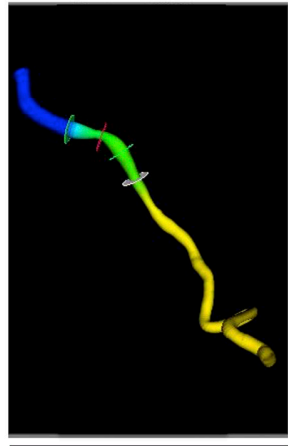

FFR 0.74

cQFR 0.83

IMR 8.91

Figure 1 Representative cases in 'jump out' and 'jump in' groups. Representative cases with cQFR $\leq 0.75$ and FFR $>0.80$ ( $A$, 'jump out') and cQFR $>0.80$ and FFR $\leq 0.75$ (B, 'jump in'). The 'jump out' case of LAD lesion showed cQFR of 0.75 nevertheless FFR of 0.83. This 'jump out' lesion had high IMR. The 'jump in' case of LCx had cQFR of 0.83 nevertheless FFR of 0.74 . This jump in' lesion had low IMR. cQFR, contrast-flow quantitative flow ratio; FFR, fractional flow reserve; IMR, index of microcirculatory resistance; LAD, left anterior descending artery; LCx, left circumflex artery.

the $\chi^{2}$ test or Fisher's exact test, as appropriate. Continuous variables are expressed as mean \pm SD for normally distributed variables and as median (25th-75th percentile) for non-normally distributed variables. Analysis was performed with the Mann-Whitney U test or KruskalWallis test for variables with a non-normal distribution and with Student's t test or analysis of variance for variables with a normal distribution. Correlations between the two parameters were evaluated using linear regression analysis. Receiver operating characteristic curves were analysed to assess the best cut-off values of the cQFR to predict an FFR of $\leq 0.80$. A two-sided p-value of $<0.05$ indicated statistical significance. To assess reproducibility between cQFR and coronary flow reserve (CFR), or FFR and CFR, we calculated Cohen's kappa coefficient.

\section{RESULTS}

\section{Patient and vessel angiographic characteristics}

Among 643 lesions of 643 patients for cQFR calculations, 139 lesions were excluded from the final analysis. The reasons for exclusion were as follows: insufficient waveform quality, pressure drift, or missing/suboptimal physiological data set (56 lesions), insufficient angiograms for cQFR calculations (57 lesions in total; 27 lesions: vessel overlap and shortening, 18 lesions: suboptimal contrast flow and 12 lesions: lack of two angiographic projections $>25^{\circ}$ apart) and no fluoroscopic documentation of the wire position of FFR measurement for use as a point of cQFR calculations (26 lesions). Therefore, the final analysis included 504 lesions of 504 patients. The median FFR, CFR and IMR values were 0.81 (0.74$0.87), 2.7$ (1.8-3.7) and 19.0 (12.6-27.5), respectively. Among all 504 lesions, $67.7 \%$ of the FFR values were distributed within the intermediate range of $0.70-0.90$. Table 1 shows the baseline and clinical characteristics. Table 2 shows per-patient and per-vessel characteristics in categories divided according to FFR and cQFR. The mean age of the patients was $66.9 \pm 9.4$ years, $88(17.5 \%)$ were women and $210(41.7 \%)$ had diabetes. Among the 504 lesions, 193 (38.3\%) showed concordantly preserved results in the FFR and QFR measurements (FFR of $>0.80$ and cQFR of $>0.80$; FFR-/cQFR-), and 203 lesions $(40.3 \%)$ showed concordantly reduced results in the FFR and cQFR measurements (FFR of $\leq 0.80$ and cQFR of $\leq 0.80 ; \mathrm{FFR}+/ \mathrm{cQFR}+$ ). Among 108 (21.4\%) discordant results, 73 lesions $(14.5 \%)$ showed a preserved FFR and decreased cQFR (FFR of $>0.80$ and cQFR of $\leq 0.80$; FFR-/ cQFR+), and 35 lesions $(6.9 \%)$ showed a reduced FFR and preserved cQFR (FFR of $\leq 0.80$ and $c Q F R$ of $>0.80$; FFR+/cQFR-) (figure 2). When the total cohort was divided into a concordant group (396 lesions, 78.6\%) and discordant group (108 lesions, 21.4\%), no significant difference in clinical or physiological characteristics was found between these two groups. The diagnostic sensitivity, specificity, accuracy, positive predictive value and negative predictive value of the cQFR for predicting an FFR of $\leq 0.80$ were $85.3 \%, 72.6 \%, 78.6 \%, 73.6 \%$ and $84.6 \%$, respectively. The area under the receiver operating characteristic curve using the cut-off threshold of $\leq 0.80$ for the FFR was 0.84 (95\% CI, 0.81 to 0.88 ) for the cQFR. Cohen's kappa coefficient between FFR and cQFR categorisation was moderate $(0.57, \mathrm{p}<0.001)$. The linear correlations between FFR/CFR and cQFR/CFR were similar $(\mathrm{R}=0.32, \mathrm{p}<0.01$ and $\mathrm{R}=0.35, \mathrm{p}<0.01$, respectively $)$.

\section{Characteristics of FFR+/cQFR- lesions}

FFR+/cQFR- lesions showed significantly milder anatomical stenosis severity than FFR+/cQFR+ lesions (area stenosis: $59.2 \%$ vs $75.3 \%$, respectively, $\mathrm{p}<0.01$; diameter stenosis: $44.6 \%$ vs $57.3 \%$, respectively, $\mathrm{p}<0.01$ ) as well as a trend for shorter lesion length (18.0 vs $20.4 \mathrm{~mm}$, respectively; $\mathrm{p}=0.15)$, similar subtended 


\begin{tabular}{|c|c|}
\hline A. Patient characteristics & Total $(n=504)$ \\
\hline Age (y) & $66.9 \pm 9.4$ \\
\hline Female gender & $416(82.5 \%)$ \\
\hline Diabetes mellitus & $210(41.7 \%)$ \\
\hline Hypertension & $352(69.8 \%)$ \\
\hline Dyslipidaemia & $316(62.7 \%)$ \\
\hline Smoking & $116(23.0 \%)$ \\
\hline eGFR (mL/min/1.73m²) & $71.9(59.5-83.7)$ \\
\hline $\mathrm{HbA1c}(\%)$ & $5.5(4.6-5.9)$ \\
\hline hs-cTnl (ng/L) & $15.0(5.3-15.0)$ \\
\hline NT-proBNP (ng/L) & $106.0(53.5-236.0)$ \\
\hline LDL cholesterol (mg/dL) & $94.0(78.0-112.0)$ \\
\hline Ejection fraction (\%) & $64(58-69)$ \\
\hline
\end{tabular}

eGFR, estimated glomerular filtration rate; $\mathrm{HbA} 1 \mathrm{c}$, glycosylated hemoglobin; hs-cTnl, high-sensitivity cardiac troponin I; LDL, lowdensity lipoprotein; NT-proBNP, N-terminal pro B-type natriuretic peptide.

\begin{tabular}{ll}
\hline B. Interrogated vessel features & Total $(\mathbf{n}=\mathbf{5 0 4})$ \\
\hline $\begin{array}{l}\text { Lesion location } \\
\text { LAD }\end{array}$ & $348(69.0 \%)$ \\
\hline LCX & $56(11.1 \%)$ \\
\hline RCA & $100(19.8 \%)$ \\
\hline Three-dimensional QCA & \\
\hline RD (mm) & $2.8(2.5-3.1)$ \\
\hline MLD (mm) & $1.4(1.1-1.7)$ \\
\hline Diameter stenosis (\%) & $49.6 \pm 12.0$ \\
\hline Area stenosis (\%) & $66.7(55.6-75.3)$ \\
\hline Lesion length (mm) & $18.4(13.1-24.0)$ \\
\hline fQFR & $0.78(0.72-0.87)$ \\
\hline CQFR & $0.79(0.74-0.88)$ \\
\hline Invasive physiological indices & \\
\hline FFR & $0.81(0.74-0.87)$ \\
\hline CFR & $2.7(1.8-3.7)$ \\
\hline IMR & $20.1(13.4-30.0)$ \\
\hline Corrected IMR & $19.0(12.6-27.5)$ \\
\hline Baseline, Tmn (s) & $0.84(0.59-1.18)$ \\
\hline Hyperaemic, Tmn (s) & $0.30(0.21-0.46)$ \\
\hline Duke jeopardy score & $2(2-6)$ \\
\hline
\end{tabular}

Variables are expressed as $\mathrm{n}(\%)$, median (IQR) or mean \pm SD. IMR correction was performed using Yong's formula.

CFR, coronary flow reserve; cQFR, contrast-flow quantitative flow ratio; FFR, fractional flow reserve; fQFR, fixed-flow quantitative flow ratio; IMR, index of microcirculatory resistance; LAD, left anterior descending artery; LCx, left circumflex artery; MLD, minimal lumen diameter; QCA, quantitative coronary angiography; $\mathrm{RCA}$, right coronary artery; $\mathrm{RD}$, reference diameter; Tmn, mean transit time. myocardial mass as indicated by the Duke jeopardy score ( 4.0 vs 4.0 , respectively; $\mathrm{p}=0.46$ ), significantly higher hyperaemic coronary flow velocity represented by a shorter mean transit time (Tmn) $(0.22$ vs 0.36 , respectively; $\mathrm{p}<0.01$ ), and a lower IMR (13.1 vs 18.8 , respectively; $\mathrm{p}=0.011$ ).

\section{Characteristics of FFR-/cQFR+ lesions}

FFR-/CQFR+ lesions showed significantly milder anatomical stenosis severity than FFR+/cQFR+ lesions (area stenosis: $71.1 \%$ vs $75.3 \%$, respectively, $\mathrm{p}=0.011$; diameter stenosis: $53.8 \%$ vs $57.3 \%$, respectively, $\mathrm{p}=0.034$ ), similar subtended myocardial mass fraction and physiological indices including coronary flow velocity represented as the inverse of Tmn.

\section{Comparison between FFR+/cQFR- and FFR-/cQFR+ lesions}

FFR-/cQFR+ lesions showed a significantly greater anatomical stenosis severity (area stenosis: $71.1 \%$ vs $59.2 \%$, respectively, $\mathrm{p}<0.01$ ), a significantly lower coronary flow velocity (Tmn: 0.33 vs 0.22 , respectively; $p=0.023$ ), and a significantly higher IMR (22.1 vs 13.1, respectively; $\mathrm{p}<0.001)$ than FFR+/cQFR- lesions. Figure 3 shows the linear correlation between the difference in the FFR and cQFR measured as a function of the anatomical stenosis severity and the physiological and subtended myocardial mass indices. According to the current cQFR calculation algorithm, an increasing anatomical stenosis severity represented by the area stenosis or diameter stenosis was associated with increasing magnitudes of the difference between the FFR and cQFR (FFR >cQFR). Similarly, a decreasing coronary flow velocity (elongation of Tmn) was associated with increasing magnitudes of the difference between the FFR and cQFR. Increasing IMR values were associated with an increasing difference between the FFR and cQFR $(\mathrm{R}=0.19, \mathrm{p}<0.01)$. In this cohort, no significant linear relationship was detected between the FFR and IMR $(\mathrm{R}=0.01, \mathrm{p}=0.82)$, while a significant although weak relationship was found between the cQFR and IMR $(R=0.15, \mathrm{p}<0.01)$. Figure 4 shows the relationship between the Duke jeopardy score and FFR or cQFR. Both measures showed a significant relationship with the subtended cardiac mass (FFR: $\mathrm{R}=0.41, \mathrm{p}<0.01$ and $\mathrm{cQFR}$ : $\mathrm{R}=0.41, \mathrm{p}<0.01$ ), while no significant trend in the difference between the two measures was detected according to the increasing subtended cardiac mass.

In total, 31 territories (6.2\%; 'jump out' group) had an FFR above the upper limit of the grey zone $(>0.80)$ and a cQFR below the lower limit $(\leq 0.75)$. In contrast, five territories (1.0\%; 'jump in' group) exhibited opposite results (FFR of $\leq 0.75$ and cQFR of $>0.80$ ). The clinical and physiological findings of these territories are shown in table 3 . The territories in the 'jump out' group showed significantly higher IMR values, lower coronary flow (elongation of Tmn) and longer lesion length $(p<0.01$ for all) than the territories in the 'jump in' group. The anatomical stenosis severity and subtended myocardial mass were similar between the groups. 
Coronary artery disease

Table 2 Patient and lesion characteristics in categories divided according to FFR and cQFR

FFR $>0.80 / c Q F R \leq 0.80$ FFR $>0.80 / c Q 8 F>0.80 /$

FFR $\leq 0.80 / c Q F R>0.80 P$ value

\begin{tabular}{|c|c|c|c|c|c|}
\hline \multicolumn{6}{|l|}{ Demographics } \\
\hline Age (y) & $68.4 \pm 9.2$ & $67.5 \pm 9.0$ & $66.5 \pm 9.6$ & $63.2 \pm 9.9$ & 0.039 \\
\hline Male gender & $61(83.6 \%)$ & $152(78.8 \%)$ & $172(84.8 \%)$ & $31(88.6 \%)$ & 0.35 \\
\hline Hypertension & $53(72.6 \%)$ & $138(71.5 \%)$ & $139(68.5 \%)$ & $22(62.9 \%)$ & 0.67 \\
\hline Dyslipidaemia & $44(60.3 \%)$ & $120(62.2 \%)$ & $128(63.1 \%)$ & $24(68.6 \%)$ & 0.87 \\
\hline \multicolumn{6}{|l|}{ Laboratory data } \\
\hline eGFR $\left(\mathrm{mL} / \mathrm{min} / 1.73 \mathrm{~m}^{2}\right)$ & $71.2(59.0-79.7)$ & $70.9(59.5-85.0)$ & $73.0(59.6-83.6)$ & $73.6(62.3-83.9)$ & 0.83 \\
\hline $\mathrm{HbA1c}(\%)$ & $5.8(5.5-6.6)$ & $5.9(5.5-6.8)$ & $5.8(5.5-6.4)$ & $6.2(5.5-6.8)$ & 0.15 \\
\hline hs-cTnl (ng/L) & $15.0(5.0-15.0)$ & $15.0(4.0-15.0)$ & $15.0(8.0-15.0)$ & $15.0(5.0-15.0)$ & 0.08 \\
\hline NT-proBNP (ng/L) & $75.0(21.0-145.0)$ & $115.5(54.0-231.3)$ & $97.0(48.0-228.3)$ & $63.0(39.5-203.5)$ & 0.041 \\
\hline LAD & $44(60.3 \%)$ & $124(64.3 \%)$ & 149 (73.4\%) & $31(88.6 \%)$ & \\
\hline LCx & $8(11.0 \%)$ & $27(14.0 \%)$ & $19(9.4 \%)$ & $2(5.7 \%)$ & 0.81 \\
\hline $\mathrm{RCA}$ & $21(28.8 \%)$ & $42(21.8 \%)$ & $35(17.2 \%)$ & $2(5.7 \%)$ & \\
\hline \multicolumn{6}{|l|}{ Three-dementional QCA } \\
\hline $\mathrm{RD}(\mathrm{mm})$ & $3.0(2.7-3.3)$ & $2.8(2.5-3.2)$ & $2.8(2.5-3.1)$ & $2.8(2.5-3.2)$ & 0.21 \\
\hline MLD (mm) & $1.4(1.1-1.6)$ & $1.6(1.4-1.9)$ & $1.2(0.9-1.4)$ & $1.5(1.1-1.6)$ & $<0.001$ \\
\hline Diameter stenosis (\%) & $53.8 \pm 7.7$ & $40.9 \pm 9.3$ & $57.3 \pm 10.1$ & $44.6 \pm 7.9$ & $<0.001$ \\
\hline Area stenosis (\%) & $71.1(64.2-75.6)$ & $56.8(45.6-65.1)$ & $75.3(67.3-81.8)$ & $59.2(52.4-63.9)$ & $<0.001$ \\
\hline IMR & $23.0(14.8-33.5)$ & $20.0(13.3-28.8)$ & $21.1(13.9-32.1)$ & $13.9(13.0-20.3)$ & $<0.001$ \\
\hline Corrected IMR & $22.1(14.8-32.6)$ & $19.4(13.0-28.4)$ & $18.8(12.3-27.2)$ & $13.1(12.0-18.9)$ & $<0.001$ \\
\hline Baseline, Tmn (s) & $0.85(0.61-1.11)$ & $0.89(0.58-1.31)$ & $0.83(0.60-1.14)$ & $0.73(0.49-1.04)$ & 0.26 \\
\hline Hyperaemic, Tmn (s) & $0.33(0.21-0.49)$ & $0.27(0.18-0.39)$ & $0.36(0.23-0.56)$ & $0.22(0.20-0.29)$ & $<0.001$ \\
\hline Duke jeopardy score & $2(0-6)$ & $2(0-4)$ & $4(2-6)$ & $4(2-4)$ & $<0.001$ \\
\hline
\end{tabular}

Variables are expressed as $\mathrm{n}(\%)$, median (IQR) or mean \pm SD.

Baseline and angiographic characteristics in four quadrants were divided based on an FFR of 0.80 and cQFR of 0.80 .

CFR, coronary flow reserve; cQFR, contrast-flow quantitative flow ratio; eGFR, estimated glomerular filtration rate; FFR, fractional flow reserve; fQFR, fixed-flow quantitative flow ratio; HbA1c, glycosylated hemoglobin; hs-cTnl, high-sensitivity cardiac troponin I; IMR, index of microcirculatory resistance; LAD, left anterior descending artery; LCx, left circumflex artery; LDL, low-density lipoprotein; MLD, minimal lumen diameter; NT-proBNP, N-terminal pro B-type natriuretic peptide; QCA, quantitative coronary angiography; RCA, right coronary artery; $\mathrm{RD}$, reference diameter; $\mathrm{Tmn}$, mean transit time.

\section{The difference of diagnostic accuracy of cQFR between cQFR+ and cQFR- lesions stratified by IMR}

When categorising 228 cQFR- lesions into two subgroups according to the reported increased IMR threshold value of $25^{15}$ (low IMR group; 165 lesions, high IMR group; 63 lesions), the diagnostic accuracy of cQFR in low IMR group was significantly lower than that in high IMR group (high IMR; $96.8 \%$ and low IMR; $80.0 \%, \mathrm{p}<0.01$ ) (figure 5C). In contrast, when $276 \mathrm{cQFR}+$ lesions were categorised into two subgroups according to the IMR value of 25 (low IMR group; 184 lesions, high IMR group; 92 lesions), high IMR group was associated with significantly lower diagnostic accuracy of cQFR than in low IMR group (high IMR; $63.0 \%$ and low IMR; $75.8 \%$, 
cQFR 0.8

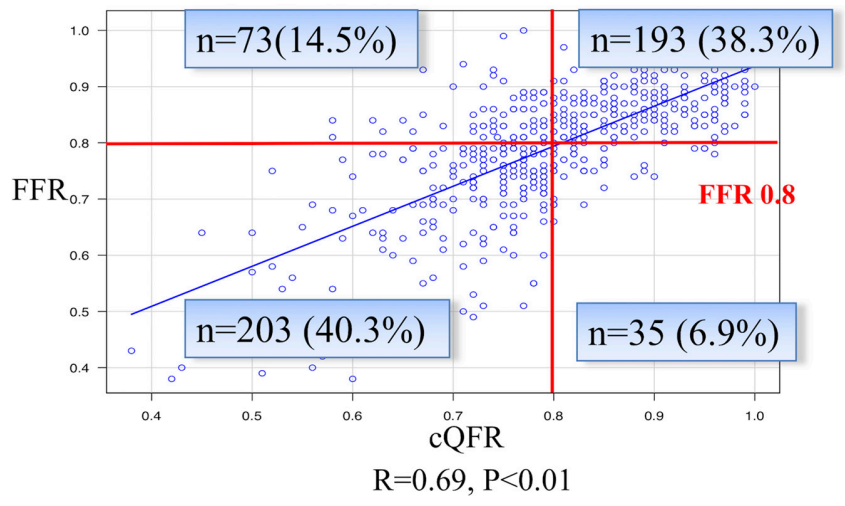

Figure 2 Agreement between FFR and cQFR. Scatter plot showing linear correlation between the FFR and CQFR. A good correlation was observed between the FFR and cQFR. However, among 504 lesions, 108 (21.4\%) showed discordant results in the FFR and CQFR measurements when the threshold of 0.80 was used. CQFR, contrast-flow quantitative flow ratio; FFR, fractional flow reserve.

$\mathrm{p}<0.01$ ) (figure 5D). In any subsets of these categorisations, cQFR consistently showed better diagnostic performance than angiographic assessments (cQFR-: 63.8\%, cQFR+: $73.2 \%$, cQFR-/high IMR: $82.5 \%$, cQFR-/low IMR: $69.7 \%$, cQFR+/high IMR: $56.5 \%$, cQFR+/low IMR: $67.4 \%$ ) (online supplementary file figure 1 ).

\section{DISCUSSION}

The important findings of the present study are as follows. First, in the present cohort of patients with angiographically intermediate stenoses, discordance in the functional stenosis classification between FFR and cQFR was observed in $21.4 \%$ (108/504). Second, FFR+/cQFRlesions showed significantly lower hyperaemic microvascular resistance, higher coronary flow velocity and significantly milder anatomical stenosis severity compared with FFR-/cQFR+ lesions, indicating a possibility of lesions similar to non-flow-limiting stenoses. Third, in the current cQFR calculation algorithm, increasing anatomical stenosis severity was associated with a greater difference between the FFR and cQFR (FFR >cQFR). Similarly, decreasing coronary flow velocity was associated with a greater difference between the FFR and cQFR (FFR $>$ CQFR). Fourth, no significant linear relationship was detected between the FFR and IMR, while a significant although weak relationship was found between the cQFR and IMR. Increasing IMR values were associated with an increasing difference between the FFR and cQFR (FFR $>$ CQFR). Fifth, both measures showed a significant relationship with the subtended cardiac mass, while no significant difference was detected in relation to an increasing subtended cardiac mass. Microvascular function bidirectionally influence cQFR diagnostic accuracy, indicating that decreased hyperaemic microvascular resistance lowered diagnostic performance of cQFR in cQFRlesions, and increased microvascular resistance worsened
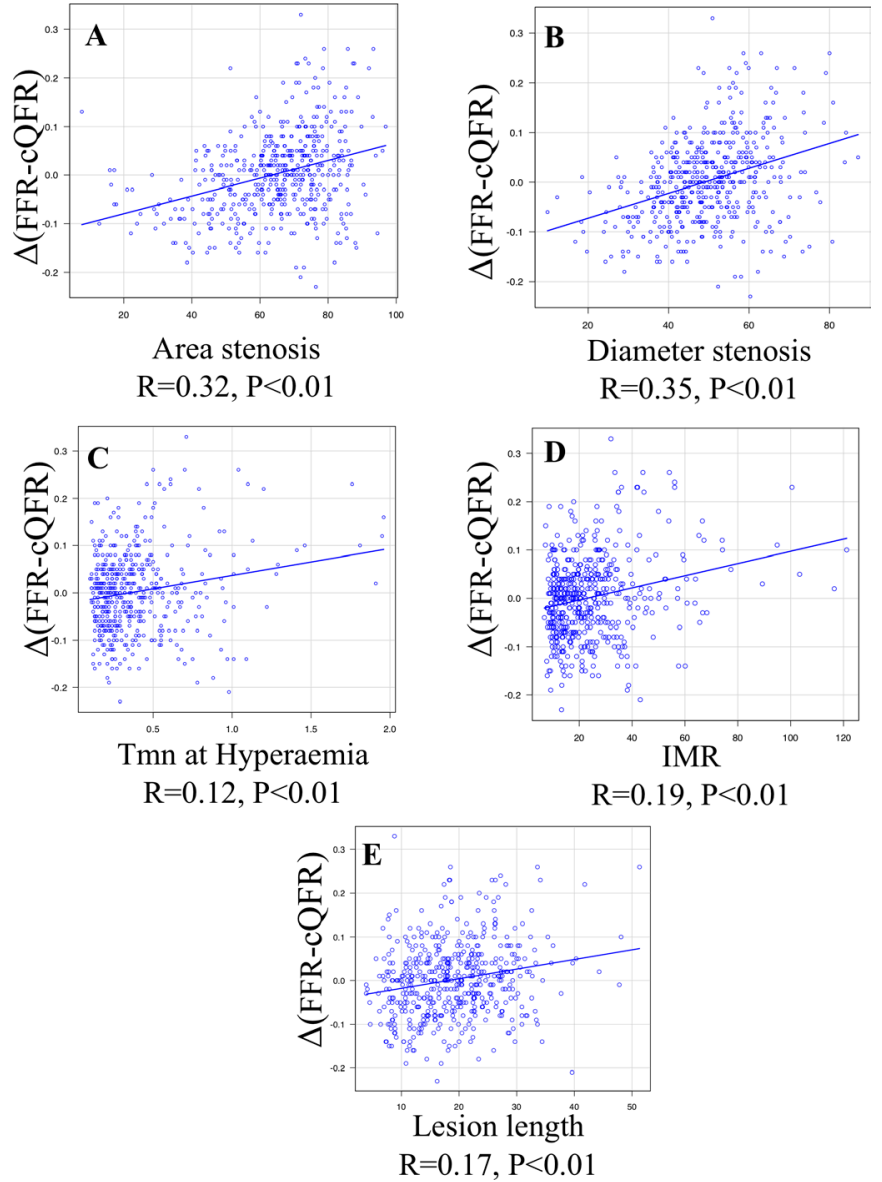

Figure 3 Linear correlation between difference in FFR/ CQFR and anatomical stenosis severity and physiological and subtended myocardial mass indices. Scatter plot showing linear relationships between the difference in the FFR and CQFR and area stenosis (A), diameter stenosis (B), Tmn at hyperaemia (C), IMR (D) and lesion length (E). A significant association was documented in the FFR-cQFR difference and area stenosis, diameter stenosis, Tmn at hyperaemia, IMR and lesion length (all $p<0.01$ ). cQFR, contrast-flow quantitative flow ratio; FFR, fractional flow reserve; IMR, index of microcirculatory resistance.

accuracy in cQFR+ lesions. Finally, in 36 lesions $(7.1 \%)$, which corresponded to about one-third of discordant functional assessment between FFR and cQFR in the present cohort, each measure jumped over the classification beyond the grey zone $(0.75-0.80)$.

Our results suggest that the cQFR may be influenced more by increasing anatomical stenosis severity and microvascular function compared with the FFR. Since the flow rate represented by the TIMI frame count was taken into consideration in the integrated calculation algorithm, the value of cQFR decreased as the hyperaemic microvascular resistance increased. This may be an important feature of the cQFR in which coronary flow is integrated in the estimation of functional stenosis severity as well as three-dimensional anatomical stenosis severity in comparison with the FFR. Moreover, our results clearly indicate that the CQFR was associated with microvascular function as represented by the IMR. This 


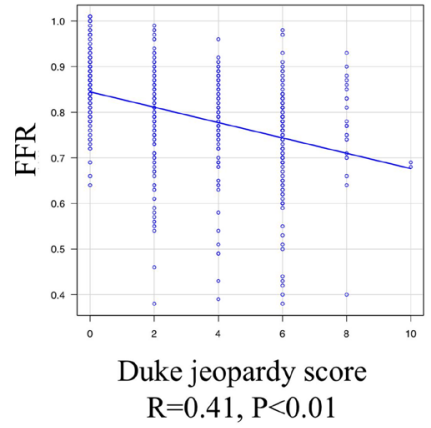

C

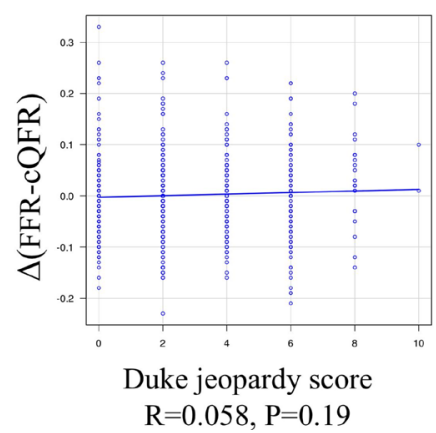

Figure 4 Linear correlation between Duke jeopardy score and FFR, cQFR and difference between FFR and cQFR. Scatter plot showing liner relationship between Duke jeopardy score and FFR (A), cQFR (B) and the difference between FFR and CQFR (C). FFR and CQFR showed significant relationships with subtended cardiac mass, while FFR-cQFR difference was not associated with the jeopardised mass. cQFR, contrast-flow quantitative flow ratio; FFR, fractional flow reserve.

is in contrast to the FFR, which reportedly has no significant relationship with the IMR, as was also shown in our study. ${ }^{16}$ Interestingly, however, unlike the difference in the relationships of the CFR with the FFR and iFR, the relationship between the cQFR and CFR was similar to that between the FFR and CFR $(\mathrm{R}=0.41, \mathrm{p}<0.01$ and $\mathrm{R}=0.41, \mathrm{p}<0.01$, respectively). ${ }^{17}$ Recently, Mejia-Renteria and Escaned et al reported that patients with coronary microvascular dysfunction showed significantly lower diagnostic performance of cQFR, compared with those without microvascular dysfunction. ${ }^{10}$ Our findings are partly in line with their results, indicating microvascular dysfunction (high IMR) lowered diagnostic performance of cQFR. We further demonstrated that decreased hyperaemic microvascular resistance represented by lower IMR similarly worsened classification agreement between FFR and cQFR. Therefore, our results extended their findings and showed that microvascular function represented by IMR bidirectionally influence cQFR diagnostic accuracy with FFR as a reference (figure $5 \mathrm{C}$ and $\mathrm{D}$ ). Furthermore, in the present study, a significant relationship between myocardial mass and cQFR was documented. Importantly, we found that 'jump out' and 'jump in' groups, which jumped beyond the grey zone $(0.75-0.80)$ into the opposite decision-making zone between FFR-guided and cQFR-guided decision-makings, existed in a nonnegligible portion of patients.

We found that increasing IMR values were associated with an increasing difference between FFR and cQFR. Furthermore, our results indicated that an increasing anatomical stenosis severity represented by the area stenosis or diameter stenosis was associated with increasing magnitudes of the difference between the FFR and cQFR (FFR $>$ CQFR), while both measures similarly showed a significant relationship with the subtended cardiac mass. Interestingly, no significant trend in the difference between the two measures was detected according to the increasing subtended cardiac mass. Of note, a decreasing coronary flow velocity (elongation of Tmn) was associated with increasing magnitudes of the difference between the FFR and cQFR, leading to the trend for FFR-/cQFR+ and lower CFR. Therefore, cQFR may be more dependent on the stenosis severity than invasive FFR in case of significant microvascular disease. Considering this basis, in territory of significant microvascular disease but with measurable epicardial coronary anatomical stenosis, the likelihood of demonstrating FFR $-/$ CQFR+ might be higher than that of FFR+/cQFR- . Conversely, in territory of well-preserved vasodilatory capacity, represented by shorter hyperaemic Tmn and low IMR, induction of hyperaemia resulted in higher pressure gradient even in the milder stenosis and resulted in FFR+. In contrast, cQFR cannot fully account patient-specific coronary flow, and therefore, the likelihood of demonstrating FFR+/cQFR- lesions might be higher than that of FFR-/cQFR+. This is, at least, one of the reasons that the diagnostic performance of $\mathrm{CQFR}$ and the agreement with invasive FFR were different depending on the IMR values. When considering the prognostic importance of CFR and IMR in FFR-negative lesions, territories with FFR-/cQFR+ lesions might indicate high-risk group for future adverse cardiac events due to severe microvascular dysfunction and/or diffuse lesion with slow coronary flow even if these lesions may be deferred on the basis of FFR-guided decision-making. ${ }^{18}$ In addition, considering higher stenosis severity in FFR-/cQFR+ lesions, plaque burden would be high in these lesions.

Notably, the present study showed that both measures exhibited a 'jumped-out' functional classification beyond the grey zone in a non-negligible proportion of lesions ( $7.1 \%$ in the total cohort). The clinical population indicated for FFR-guided decision-making is predominantly based on the presence of intermediate lesions with FFR values showing a unimodal concentration close to the cut-off value of 0.80 or within the grey zone. In addition, measurement errors including technical imprecision and bias such as pressure drift can have an important effect on clinical decision-making regarding revascularisation, particularly in cases near the decision cut-off or close to the boundaries of the grey zone, and may affect the classification of patients for therapeutic management. ${ }^{14} 19$ 
Table 3 Characteristics of 'jump in' and 'jump out' groups

\begin{tabular}{|c|c|c|c|}
\hline & Jump out ( $n=31)$ & Jump in $(n=5)$ & $P$ value \\
\hline Lesion location & & & 0.078 \\
\hline LAD & $16(51.6 \%)$ & $3(50.0 \%)$ & \\
\hline $\mathrm{LCx}$ & $3(9.7 \%)$ & $2(40.0 \%)$ & \\
\hline RCA & $12(38.7 \%)$ & $0(0 \%)$ & \\
\hline fQFR & $0.71(0.64-0.73)$ & $0.84(0.84-0.89)$ & 0.0017 \\
\hline cQFR & $0.73(0.68-0.74)$ & $0.85(0.83-0.88)$ & $<0.001$ \\
\hline FFR & $0.85(0.83-0.91)$ & $0.74(0.72-0.74)$ & $<0.001$ \\
\hline CFR & $2.2(1.6-3.0)$ & $3.5(3.4-4.2)$ & 0.13 \\
\hline IMR & $28.9(17.9-43.0)$ & $13.4(12.9-14.1)$ & 0.019 \\
\hline Corrected IMR & $28.3(17.5-41.7)$ & $12.3(11.7-12.9)$ & $<0.001$ \\
\hline Baseline, Tmn (s) & $0.58(0.20-0.84)$ & $0.72(0.46-0.96)$ & 0.65 \\
\hline Hyperaemic, Tmn (s) & $0.26(0.13-0.49)$ & $0.21(0.19-0.24)$ & $<0.001$ \\
\hline Diameter stenosis (\%) & $73.4 \pm 5.3$ & $46.2 \pm 7.7$ & 0.21 \\
\hline Area stenosis (\%) & $56.5(52.7-59.6)$ & $59.4(58.3-62.5)$ & 0.095 \\
\hline Lesion length (mm) & $23.5(18.6-26.3)$ & $17.8(17.4-18.1)$ & 0.0025 \\
\hline $\operatorname{MLD}(\mathrm{mm})$ & $1.40(1.33-1.58)$ & $1.10(1.10-1.25)$ & 0.97 \\
\hline $\mathrm{RD}(\mathrm{mm})$ & $2.9(2.7-3.2)$ & $2.9(2.5-3.2)$ & 1 \\
\hline Duke jeopardy score & $2(2-6)$ & $4(2-4)$ & 1 \\
\hline
\end{tabular}

Variables are expressed as $\mathrm{n}(\%)$, median (IQR) or mean \pm SD.

CFR, coronary flow reserve; cQFR, contrast-flow quantitative flow ratio; FFR, fractional flow reserve; fQFR, fixed-flow quantitative flow ratio; IMR, index of microcirculatory resistance; IMR, index of microvascular resistance; LAD, left anterior descending artery; LCx, left circumflex artery; MLD, minimal lumen diameter; RCA, right coronary artery; RD, reference diameter; Tmn, mean transit time.

We demonstrated that in $7.1 \%$ of lesions, which corresponded to about one-third of discordant functional assessment between FFR and cQFR in the present cohort, one metric was above the upper limit of the grey zone and the other was below the lower limit, which might raise a critical concern when using the CQFR as an interchangeable marker of the FFR during revascularisation decision-making or therapeutic management.

The FFR is determined both by the anatomical stenosis severity and by the amount of subtended myocardial mass distal to the lesion. ${ }^{20}$ Our results are in line with previous studies and showed that the FFR and cQFR were both significantly and similarly associated with the jeopardised myocardium, suggesting that the amount of myocardial mass subtended by a coronary stenosis is appropriately incorporated into the cQFR algorithm similar to the FFR.

The QFR computation was recently developed and validated to estimate the FFR value without pressure wire and pharmacologically induced hyperaemia. These are favourable aspects of QFR in the clinical practice. In addition, QFR measurements could shorten the procedure time and reduce wire-related cost and risks. However, discrepancy between the FFR-guided and cQFR-guided decision-making was documented in $20 \%$ of patients when the cut-off value of 0.80 was applied in the present study. Outcome date are still scarce, and further studies should clarify if cQFR-based decision-making is superior or non-inferior to FFR-based revascularisation decision-making. In addition, we could use QFR as a part of hybrid approach for decision-making without wirebased functional measurements.

As mentioned above, our study demonstrated that, in $36(7.1 \%)$ lesions, in which the functional assessment jumped beyond the grey zone $(0.75-0.80)$ into the opposite decision-making zones. This observation would be important, whereas there could be a potential to take full advantage of this feature to identify patients at high risk with slow coronary flow and high microvascular resistance that will lead to worse prognosis, even though these lesions have FFR $>0.80$.

Lesions with a discordant FFR and cQFR classification may need to be considered as a different functional and anatomical entity, not merely as an imprecision or technical error. Further studies are needed to elucidate whether these discordances can provide prognostic information.

\section{Study limitations}

The results of the present study should be interpreted with some limitations. This was a retrospective study and its inherent selection bias and limitations regarding the retrospective analysis of stored angiograms may exist. This might have also affected the accuracy of cQFR in the current study. Although care was taken to match the measurement points of the FFR and cQFR, small differences in location may have occurred in some cases. No 
A

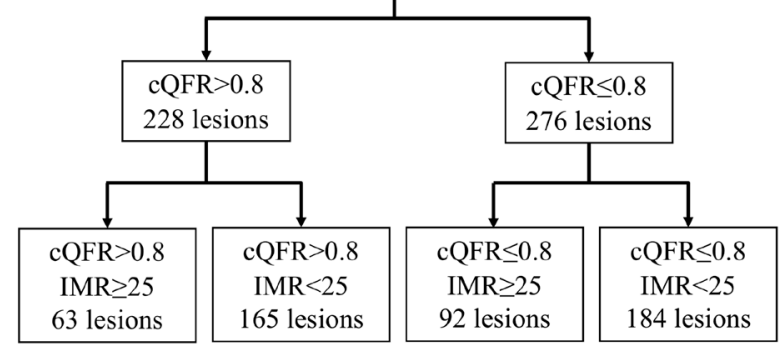

B

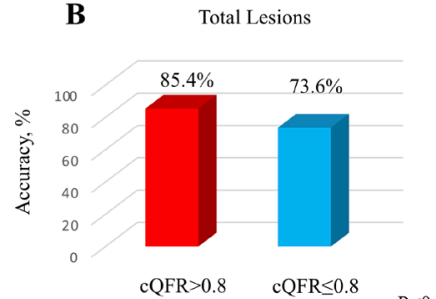

D

cQFR + lesions $(\mathrm{cQFR} \leq 0.80)$

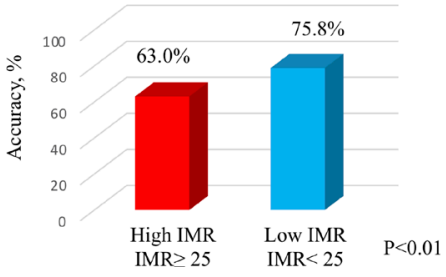

Figure 5 The difference in diagnostic accuracy in lesions classified by cQFR and IMR. (A) A total cohort was classified by cQFR and IMR. (B) The agreement between cQFR and FFR was better in CQFR- lesions. (C) The diagnostic accuracy was better in lesions with increased microvascular resistance in cQFR- lesions. (D) In cQFR+ lesions, increased microvascular resistance was associated with lower diagnostic accuracy. cQFR, contrast-flow quantitative flow ratio; IMR, index of microcirculatory resistance.

outcome data were obtained to assess the clinical value of the cQFR. Further studies are required to elucidate whether the cQFR may provide a prognostic benefit compared with angiographic guidance or whether cQFRguided revascularisation decision-making shows noninferiority to FFR guidance.

\section{CONCLUSIONS}

Microvascular function and the anatomical stenosis severity were significantly associated with discordant FFR and CQFR values, while the subtended myocardial mass showed no impact on the difference between the FFR and cQFR. When using the FFR as a reference, the cQFR overestimated functional stenosis severity with increasing microvascular resistance and anatomical stenosis severity. Microvascular function bidirectionally influenced classification agreement between FFR and cQFR according to functional significance of lesions. Care should be taken when the cQFR is used for revascularisation decisionmaking because the cQFR and FFR values may result in completely different decisions beyond the grey zone in more than one-third of discordant classifications between two measures.

Acknowledgements We thank all the physicians, nurses, other catheter laboratory staff members and patients involved in this study.

Contributors YKanno designed the study, and wrote the initial draft of the manuscript. TK, TS, MHo and RH contributed to analysis and interpretation of data, and assisted in the preparation of the manuscript. All other authors have contributed to data collection and interpretation, and critically reviewed the manuscript. All authors approved the final version of the manuscript, and agreed to be accountable for all aspects of the work in ensuring that questions related to the accuracy or integrity of any part of the work are appropriately investigated and resolved.

Funding The authors have not declared a specific grant for this research from any funding agency in the public, commercial or not-for-profit sectors.

Competing interests None declared.

Patient consent for publication Not required.

Provenance and peer review Not commissioned; externally peer reviewed.

Data availability statement Data are available upon reasonable request.

Open access This is an open access article distributed in accordance with the Creative Commons Attribution Non Commercial (CC BY-NC 4.0) license, which permits others to distribute, remix, adapt, build upon this work non-commercially, and license their derivative works on different terms, provided the original work is properly cited, appropriate credit is given, any changes made indicated, and the use is non-commercial. See: http://creativecommons.org/licenses/by-nc/4.0/.

ORCID iDs

Masahiro Hoshino http://orcid.org/0000-0003-3661-1914

Taishi Yonetsu http://orcid.org/0000-0002-1798-5008

Tsunekazu Kakuta http://orcid.org/0000-0002-6283-5412

\section{REFERENCES}

1 Pijls NHJ, Tanaka N, Fearon WF. Functional assessment of coronary stenoses: can we live without it? Eur Heart J 2013;34:1335-44.

2 De Bruyne B, Fearon WF, Pijls NHJ, et al. Fractional flow reserveguided PCl for stable coronary artery disease. N Engl J Med 2014;371:1208-17.

3 Toth GG, Toth B, Johnson NP, et al. Revascularization decisions in patients with stable angina and intermediate lesions: results of the International survey on interventional strategy. Circ Cardiovasc Interv 2014;7:751-9.

4 Pijls NHJ, Sels J-WEM. Functional measurement of coronary stenosis. J Am Coll Cardiol 2012;59:1045-57.

5 Tu S, Westra J, Yang J, et al. Diagnostic Accuracy of Fast Computational Approaches to Derive Fractional Flow Reserve From Diagnostic Coronary Angiography: The International Multicenter FAVOR Pilot Study. JACC Cardiovasc Interv 2016;9:2024-35.

6 Tu S, Barbato E, Köszegi Z, et al. Fractional flow reserve calculation from 3-dimensional quantitative coronary angiography and TIMI frame count: a fast computer model to quantify the functional significance of moderately obstructed coronary arteries. JACC Cardiovasc Interv 2014;7:768-77.

7 Xu B, Tu S, Qiao S, et al. Diagnostic Accuracy of Angiography-Based Quantitative Flow Ratio Measurements for Online Assessment of Coronary Stenosis. J Am Coll Cardiol 2017;70:3077-87.

8 Smit JM, Koning G, van Rosendael AR, et al. Relationship between coronary Contrast-Flow quantitative flow ratio and myocardial ischemia assessed by SPECT Mpi. Eur J Nucl Med Mol Imaging 2017;44:1888-96.

9 Westra J, Tu S, Winther S, et al. Evaluation of coronary artery stenosis by quantitative flow ratio during invasive coronary angiography: the WIFI II study (Wire-Free functional imaging II). Circ Cardiovasc Imaging 2018;11:e007107.

10 Mejía-Rentería H, Lee JM, Lauri F, et al. Influence of microcirculatory dysfunction on Angiography-Based functional assessment of coronary stenoses. JACC Cardiovasc Interv 2018;11:741-53.

11 Echavarría-Pinto M, van de Hoef TP, Nijjer S, et al. Influence of the amount of myocardium subtended to a coronary stenosis on the index of microcirculatory resistance. Implications for the invasive assessment of microcirculatory function in ischaemic heart disease. Eurolntervention 2017;13:944-52. 
12 Yonetsu T, Murai T, Kanaji Y, et al. Significance of microvascular function in Visual-Functional mismatch between invasive coronary angiography and fractional flow reserve. J Am Heart Assoc 2017;6. doi:10.1161/JAHA.117.005916

$13 \mathrm{Ng}$ MKC, Yeung AC, Fearon WF. Invasive assessment of the coronary microcirculation: superior reproducibility and less hemodynamic dependence of index of microcirculatory resistance compared with coronary flow reserve. Circulation 2006;113:2054-61.

14 Wakasa N, Kuramochi T, Mihashi N, et al. Impact of pressure signal drift on fractional flow Reserve-Based decision-making for patients with intermediate coronary artery stenosis. Circ J 2016;80:1812-9.

15 Liu A, Wijesurendra RS, Liu JM, et al. Diagnosis of microvascular angina using cardiac magnetic resonance. J Am Coll Cardiol 2018;71:969-79.

16 Yong ASC, Ho M, Shah MG, et al. Coronary microcirculatory resistance is independent of epicardial stenosis. Circ Cardiovasc Interv 2012;5:103-8.
17 Cook CM, Jeremias A, Petraco R, et al. Fractional Flow Reserve/ Instantaneous Wave-Free Ratio Discordance in Angiographically Intermediate Coronary Stenoses: An Analysis Using DopplerDerived Coronary Flow Measurements. JACC Cardiovasc Interv 2017;10:2514-24.

18 Lee JM, Jung J-H, Hwang D, et al. Coronary flow reserve and microcirculatory resistance in patients with intermediate coronary stenosis. J Am Coll Cardiol 2016;67:1158-69.

19 Pijls $\mathrm{NH}$, Bruyne BD. Fractional flow reserve, coronary pressure wires, and drift. Circ J 2016;80:1704-6.

$20 \mathrm{Kim}$ HY, Lim H-S, Doh J-H, et al. Physiological Severity of Coronary Artery Stenosis Depends on the Amount of Myocardial Mass Subtended by the Coronary Artery. JACC Cardiovasc Interv 2016;9:1548-60. 